\title{
Una estrategia de respuesta rápida en desastres
}

\author{
Daniel Pinzón-Gómez ${ }^{*}$ y Fabián Leonardo Valencia-Arévalo² \\ ${ }^{1}$ Administrador en Salud, Universidad de Antioquia, Calle 67 No. 53 - 108 Conmutador, Medellín, Antioquía, Colombia, \\ Corporación Universitaria Adventista de Colombia, Carrera $84 N^{\circ} 33 a a-01$ La Castellana, Medellín, Colombia \\ ${ }^{2}$ Docente de Ciencias Básicas Biomédicas, Universidad Pontificia Bolivariana, Carrera 43C \# 72a-173, Medellin, Antioquia, Colombia
}

\begin{abstract}
RESUMEN
Se presenta la experiencia y la práctica profesional de un equipo adventista de búsqueda y rescate (SAR, de su sigla en inglés) colombiano, que prestó su colaboración a una comunidad confesional en el municipio de Carrefour, Haití, tras el terremoto del 12 de enero de 2010.

Las acciones fueron dirigidas a las áreas de búsqueda y rescate urbano en estructuras colapsadas (BREC), con equipo liviano, manejo del trauma y saneamiento ambiental, tomando en cuenta las condiciones socioculturales de la población de emergencia, en el municipio de Carrefour, donde se puso en evidencia que la gestión previa, la planeación y la organización, así como el entrenamiento son cruciales para el éxito de estas misiones. La experiencia de este trabajo de campo puede servir como guía para organizaciones confesionales y personal de respuesta (técnicos, tecnólogos y profesionales de la salud) en caso de desastres y poblaciones desplazadas.
\end{abstract}

Palabras clave: Desastres, búsqueda y rescate, trauma, saneamiento ambiental.

\begin{abstract}
The objective of the research was to determine the level of knowledge, attitudes and practices of bioethical principles of nursing in inpatient care, the Hospital II-EsSalud Vitarte, 2011. Design Studio quantitative, exploratory and descriptive scope. Was conducted in a sample composed of 35 nurses who work in nursing care Medical Services, Surgery, Emergency and ICU (Intensive Care Unit). A questionnaire was used to measure the level of knowledge, attitudes and a scale for a guide to practical observation. Areas covered in this study were: Ethics, Ethics, Bioethics, beneficence, autonomy, confidentiality, informed consent, justice. The results were that $20 \%$ of participants have an excellent level of knowledge of bioethical principles, $45.7 \%$ have a good level, $31.4 \%$ and $2.9 \%$ regularly has a poor level. Regarding attitude levels of bioethical principles is positive $71.4 \%$ and $28.6 \%$ has a moderately positive attitude level. As for practices, $60 \%$ of participants practiced bioethical principles during patient care. Which means that most nurses, have a level of knowledge and level of attitudes of bioethical principles, good and positive respectively. Similarly, the level of practice is acceptable and required in patient care.
\end{abstract}

Keywords: Disasters, search and rescue, trauma, environmental sanitation.

*Correspondencia: pinzon.gomez@gmail.com / fableo42@hotmail.com 


\section{INTRODUCCIÓN}

Cuando ocurre un desastre, la prioridad es rescatar y socorrer a las víctimas. La evidencia ha demostrado que, en la mayoría de los casos, las acciones asistenciales, búsqueda y rescate se concentran en las primeras 72 horas, tiempo en el que se presentan la mayor cantidad de posibilidades de encontrar, evacuar y asistir a los lesionados, igualmente dicha asistencia es dispensada por las comunidades locales quienes se movilizan espontáneamente, ofreciendo las acciones de rescate más básicas hasta las respuestas en salud más sofisticadas a las que tengan acceso, razón por la cual la participación de equipos de salud y rescate extranjeros, destinados al apoyo a países afectados por un desastre, ha sido desestimulada en las últimas décadas, debido a que esta ayuda puede llegar retardada y después de determinado tiempo, son pocas las intervenciones que pueden realizarse para salvar vidas (Federación Internacional de Sociedades de la Cruz Roja y de la Media Luna Roja, 2009). De la misma manera, la experiencia ha evidenciado que estos especialistas presentan limitaciones por el desconocimiento del territorio, la cultura y el idioma, sumadas a los problemas para alojarlos y alimentarlos adecuadamente, constituyéndose en una carga adicional para el país afectado, [por lo cual es preferible que la cooperación esté enfocada al desarrollo de las capacidades locales para superar la crisis (Noji, $2000 \&$ OPS (2008).

No obstante, el evento de Haití en enero de 2010, puso en evidencia que ningún país está completamente preparado para enfrentar un desastre de gran magnitud, toda vez que la mayoría de los desastres están sujetos a problemas de desarrollo y gobernabilidad no resueltos y especialmente en Latinoamérica y el Caribe (LAC) los cuales constituyen una amenaza latente para la cual la comunidad internacional deberá estar dispuesta a responder con sentido solidario y humanitario. Siempre será necesario disponer de equipos de respuesta rápida ante desastres con equipos de despliegue rápido, solventemente equipados y con suficientes capacidades técnicas, asistenciales y logísticas acordes con las necesidades (OPS, 2010 \& Gellet, 2012).

La respuesta ante desastres, en todos los niveles, en muchas ocasiones está mediada por la excesiva improvisación, por tanto, es siempre menester una adecuada planificación, con el fin de garantizar el máximo éxito de las operaciones, evitando ser una carga al país beneficiado, garantizando la seguridad de las operaciones y siendo un alivio y auxilio próximo para los afectados. En este sentido la Secretaría del International Search and Rescue Advisory Group (INSARAG), dependencia adscrita a la Oficina del Alto Comisionado para Asuntos Humanitarios (OCHA) de las Naciones Unidas (NN.UU.), ha desarrollado guías y metodologías para acciones de USAR a nivel internacional de acogencia discrecional para los grupos de respuesta a desastres (OPS, 2012; Rodríguez A. \& Terry, 2002; INSARAG, 2012).

En este artículo se describe la experiencia de un equipo SAR adventista de Colombia dando respuesta de cooperación humanitaria al desastre en Haití, con motivo del terremoto que afectó en el año 2010 a este país, basados en el espíritu de planeación y directrices de INSARAG.

\section{Características del evento y del entorno}

El 12 de enero de 2010, a las 16.53.09, hora local, se produjo en la localidad de Léogâne cerca de Puerto Príncipe, la capital de Haití, un fuerte terremoto que alcanzó una magnitud de 7.1 en la escala sismológica de Richter. En los días siguientes se registraron réplicas de magnitudes altas $(5.9,5.5$ y 5.1), agravando el daño inicial aún más, hasta alcanzar cifras en daños a las personas, bienes, servicios y medio ambiente de manera dramática. La cifra final de muertos fue de 222.570 y de heridos en más de 300.000 , con una destrucción de la capital de alrededor del 90\% y 1.300 .000 personas sin hogar (Médiz, Cristófol, \& Navas, 2010).

Las consecuencias de este desastre agravaron las condiciones sociales, políticas, económicas, ambientales $\mathrm{y}$, en general, de salud pública que ya prevalecía antes del sismo, con la consecuente interrupción en la prestación de los servicios vitales como las comunicaciones, el transporte, de seguridad pública (policía, bomberos y cuidados prehospitalarios), la dramática y desmoralizadora pérdida de los equipamientos de salud, así como del personal asistencial que resultaron seriamente afectados, de la misma manera el ya limitado y precario recurso de atención a emergencias local se vio seriamente afectado, sin capacidad de respuesta inicial. La situación se agravó con el desplazamiento de la población a sitios más seguros o sin riesgo de colapsar que, unidos a la precaria red de servicios públicos (acueducto, alcantarillado, electricidad), la escasez de alimentos y su inadecuada manipulación detonaron fenómenos de carácter social y de salud concomitantes, con el debido hacinamiento de alojamiento temporales.

De otro lado, la Iglesia Adventista del Séptimo Día (IASD) en Haití con una alta influencia en la isla, contaba con una feligresía de 335000 feligreses, una 
universidad, dos academias, un hospital y una emisora radial. Los informes de afectación de esta comunidad fueron de: 522 adventistas muertos, entre ellos 10 pastores; 55 templos totalmente destruidas y 66 parcialmente dañados; 27,000 miembros perdieron sus hogares; cuatro escuelas primarias destruidas (Negrín, Betancour, Sánchez \& Monterrey, 2006).

Pese a la devastadora destrucción del terremoto, tanto la Universidad Adventista de Haití como el Hospital Adventista de Diquini, así como las instalaciones de la Agencia Adventista de Desarrollo y Recursos Asistenciales (ADRA), ubicados en la municipalidad de Carrefour no fueron dañados por el terremoto, constituyéndose en referentes de apoyo al desastre, especialmente por la organización y la mínima afectación de las estructuras vitales que sirvieron de soporte y contención en su área de influencia para la población afectada.

\section{Desarrollo de la experiencia}

Tras conocerse el impacto del sismo que afectara la capital de Haití, numerosos estados y agencias no gubernamentales alrededor del mundo desplegaron ayuda humanitaria de manera inmediata. El gobierno colombiano, representado por su Cancillería y la representación diplomática de Haití en Colombia, coordinaron las acciones para el envío de ayudas representadas en talento humano calificado, suministros humanitarios, así como apoyo logístico.

Una de las misiones enviadas estuvo conformada por un equipo SAR de la Corporación Grupo Adventista de Rescate y Salvamentos (GARSA), agencia humanitaria de carácter confesional, civil, jurídica, administrativa y financieramente autónoma adscrita al Sistema Nacional de Gestión del Riesgo de Desastres (SNGRD) de la república de Colombia, la cual cuenta con experiencia en manejo de desastres desde 1992.

El equipo SAR estuvo conformado por técnicos, tecnólogos y profesionales adventistas con experticia en desastres, el cual se desplazó al municipio de Carrefour, Haití, el 15 enero de 2010, con una permanencia en la zona de 10 días.

La misión pudo llevarse a cabo gracias a las gestiones que la Corporación GARSA en conjunto con la UNGRD de Colombia, las gestiones políticoadministrativas efectuadas por ADRA de Colombia y un representante del Senado de la República.

El equipo voló desde Medellín (Colombia) hasta
Puerto Príncipe (Haití), en un avión Boeing 737 de la Fuerza Aérea Colombiana (FAC), acompañado de un equipo humanitario del Gobierno Nacional que apoyaría otros frentes de trabajo. En el sitio, el equipo SAR adventista fue trasladado por una escolta militar de cascos azules de las Naciones Unidas, hacia el municipio de Carrefour, área urbana de mayor representatividad de la IASD del país afectado, donde se encuentra localizado el complejo institucional educativo, a saber, la Universidad Adventista de Haití y su respectivo colegio, junto con las industrias alimenticias y el templo adventista de la universidad, además del Hospital Adventista de Diquini, y las oficinas y bodegas estratégicas de ADRA Haití, contiguas a la universidad.

\section{Organización y acciones operativas}

La misión se organizó en grupos y frentes de trabajo:

Equipo de Rescate y Salvamento Urbano (de su sigla en inglés, USAR):

Compuesto por un líder de equipo, un líder en traducción (políglota), una técnica canica y dos técnicos USAR. Todo el personal contaba con experiencia en diversos tipos de urgencias y emergencias. La zona general de operaciones USAR se estableció en el área urbana periférica a la institucionalidad adventista, donde hasta la fecha del arribo del equipo no había tenido presencia de organismo de apoyo humanitario alguno. Las acciones se centraron en la búsqueda de sobrevivientes (humanos y/o animales), como también a la recuperación de cuerpos sin vida a través de técnicas de BREC liviano.

\section{Equipo de salud}

Compuesto por un médico con experiencia en manejo del trauma en desastres (bilingüe) y dos tecnólogos en atención prehospitalaria, igualmente experimentados en urgencias, emergencias y desastres. Este equipo se localizó en el Hospital Adventista de Diquini, en el cual emplazó una carpa hospital y dio apoyo instrumental para la adecuación del quirófano externo al edificio hospitalario.

En el momento del establecimiento se requería la atención de alrededor 200 personas con traumas de diversos niveles. Las acciones estuvieron encaminadas a apoyar el equipo médico local que se encontraba al máximo de su capacidad ocupacional ( $\sin$ relevos), muchos de ellos viviendo el drama de la pérdida de sus familiares. Se establecieron acciones que incluyeron la organización del triaje -y su diaria aplicación en pro de 
un censo epidemiológico continuo-, la estabilización del soporte vital básico y del avanzado, al igual que se apoyó las acciones terapéuticas de mayor prioridad, tales como amputaciones, lavados quirúrgicos y demás procedimientos de alta cirugía.

Posterior al trabajo realizado en este lugar, por un período aproximado de 8 días, el equipo se traslada al Hospital Universitario de la Paz para apoyar labores asistenciales en el manejo del trauma y emergencia médica, - especialmente por infarto agudo de miocardio (IAM) - estas últimas documentadas en la literatura sobre epidemiología asociadas a las condiciones de posdesastre (Negrín, 2012; Arcos, Castro \& Busto, 2002):

\section{Equipo de saneamiento básico}

Tal como lo registra la literatura, se identificó la afectación del servicio de recolección de residuos sólidos, peligrosos y similares, tras la ocurrencia del terremoto, por lo cual las acciones estuvieron destinadas a mitigar el impacto de las condiciones ambientales que pudieren afectar la salubridad en la institución hospitalaria (OPS, 2003).

El equipo estuvo compuesto por un profesional en salud pública, apoyado por un profesional políglota. Se organizan a líderes y familiares de los pacientes hospitalizados, que se encontraban refugiados en el hospital, los cuales se les orienta en el proceso de separación, clasificación y disposición de residuos sólidos. Se identificó la ausencia de protocolos para la disposición de residuos de riesgo biológico en la institución, los cuales se disponían en zonas verdes cercanas y a cielo abierto. Las acciones se encaminaron a la limpieza de los alrededores de los quirófanos temporales, como disposición de los materiales que representaban riesgo biológico, incluidos restos anatomopatológicos.

De la misma manera que lo hizo el equipo de salud, este equipo se traslada al Hospital Universitario de la Paz de Puerto Príncipe para apoyar la gestión de cadáveres y el saneamiento en el establecimiento de salud.

\section{Organización de la logística individual}

Cada profesional tenía previsto una mochila de 70 libras, que contienen dos mudas de ropa, equipo de limpieza personal, botiquín personal y equipo de supervivencia personal, carpa individual y raciones de campaña. La logística individual tiene una autonomía para 20 días, con el adicional porte de dinero en dólares.
Anexo a lo anterior, cada rescatista debía portar equipo de protección personal: casco testado, linterna frontal, monogafas, tapabocas, guantes quirúrgicos y de lona antideslizante; botas de cuero con punta metálica y rodilleras. Dadas las circunstancias climáticas del lugar, siempre se incluyó 1 a 2 litros de agua potable (llevada desde Colombia), como también protector solar. Se contó con estufa portátil de combustible sólido por cada trío de personas del equipo.

\section{Organización de la logística de equipo}

En cantidades impares, la corporación contaba con:

- Planta eléctrica.

- Canino hembra de raza Fox Retriever (1.5 años de edad), entrenada en búsqueda en escombros, junto a su respectivo guacal.

- Botiquín médico avanzado para el manejo de trauma.

- Carpa hospital.

- Lámparas reflectoras.

A pesar de las limitaciones idiomáticas que fueron sorteadas con el servicio de traducción, la afinidad confesional del equipo colombiano y el personal del Hospital Adventista de Diquini fue lo que mayormente facilitó la receptividad y la ayuda recíproca en el trabajo de equipo. Su presencia constituyó un aliciente muy esperanzador para una organización receptora de un gran porcentaje de lesionados del área afectada.

\section{Principales resultados}

En la actualidad, la Corporación GARSA, sucursal Medellín-Colombia, lleva trabajando 18 años en el ámbito de la respuesta en urgencias, emergencias y desastres, situándose como líder en la implantación de la atención prehospitalaria y las acciones de búsqueda y rescate en áreas urbanas y rurales. Su experiencia se ha fraguado en el marco de las amenazas naturales, los fenómenos tecnológicos y el conflicto armado colombiano, escenarios que le han permitido posicionarse como una agencia humanitaria con alta capacidad de respuesta, principios de liderazgo servidor y valores agregados como el apoyo espiritual es desastres, faceta sine qua non a la hora de hablar de su papel comparativo con otras entidades afines a su objeto social. La participación de la agencia posibilitó consolidar su capacidad de respuesta ante eventos de gran magnitud.

Circunstancias de desastre semejantes a las presentadas en Haití durante el terremoto de 2010, evidencian que a pesar del desestímulo por el envío de 
expertos internacionales por parte de los organismos multilaterales, demuestran la aun necesidad vigente de apoyar comunidades afectadas por desastres que, por sus limitadas condiciones de desarrollo previo, les resulta imperiosa la ayuda externa para la provisión de socorro inicial, especialmente en acciones de búsqueda, rescate y cuidados médicos urgentes. La respuesta del equipo SAR colombiano a la localidad de Carrefour, donde hay una fuerte presencial denominacional, resultó de ayuda invalorable para muchas personas cercanas al vecindario adventista.

Pocas horas después del evento sísmico, se había establecido una coordinación para la respuesta entre la UNGRD y la Corporación GARSA. La organización administrativa y logística se desarrolló exitosamente en tiempo límite.

En el terreno se identificaron puntos estratégicos para coordinar las acciones de búsqueda y rescate urbano en cuatro puntos estratégicos del área urbana de Carrefour.

Las acciones de búsqueda en escombros se realizaron de acuerdo a los patrones estandarizados para equipos USAR. Las acciones de rescate se enfatizaron en estructuras colapsadas, en todo momento apoyados por el equipo traductor.

En el hospital se organizó el equipo asistencial para optimizar las acciones de triaje y de atención de las urgencias, según prioridad se establecieron criterios de atención estandarizados que facilitaron la atención y el control de la situación frente al manejo de los lesionados. La adecuación de una carpa hospital y la habilitación de un quirófano externo posibilitó la atención del $70 \%$ los afectados durante los días que estuvo el equipo SAR en el terreno.

El apoyo médico asistencial del equipo SAR colombiano representó un alivio para el personal del Hospital Adventista de Diquini, local que se encontraba al límite de su capacidad. Las actividades se centraron en procedimientos para manejo de traumatismos en miembros inferiores, superiores y cabeza; el apoyo quirúrgico estuvo orientado a los procedimientos de amputación, apoyo en los servicios de radiología, manejo de eventos cardiovasculares, crisis hipertensiva, entre otros.

Dada la magnitud del evento, demandada de atención urgente, las limitaciones logísticas y de personal la atención fue insuficiente para impedir muertes de personas que, en otras condiciones y circunstancias, se hubieran podido evitar.

El equipo SAR de Colombia logró realizar empalme exitoso y sinérgico con un grupo de salud pública proveniente de Francia, con el cual se coordinaron las acciones que posibilitaron la continuidad de las acciones instauradas.

Las acciones de saneamiento básico posibilitaron mitigar los focos de contaminación y riesgo biológico que amenazaban la seguridad de los procedimientos quirúrgicos realizados en el hospital. Sin embargo, las limitaciones logísticas para el establecimiento de letrinas y adecuación de áreas específicas restringieron los alcances del programa.

A pesar que hubo una estrategia, no se abordó de manera integral las intervenciones de saneamiento básico con el fin de poder garantizar continuidad de estas. Se presentó resistencia por parte de los administradores del hospital en la adecuación de áreas específicas para el manejo y disposición de restos anatomopatológicos.

Dado la magnitud del evento, una vez llegados a Colombia se sometió a chequeos médicos y posteriores sesiones de apoyo psicológico.

\section{CONCLUSIONES}

Se resaltan los aspectos positivos y negativos que fueron más relevantes en este reporte con respecto al área:

Las gestiones político-administrativas entre tomadores de decisiones y el trabajo positivo posibilitó a la Corporación GARSA la opción del traslado en tiempo récord.

La larga trayectoria de la Corporación GARSA durante 18 años y su alto posicionamiento en la asistencia humanitaria en Colombia, posibilitó su inclusión entre la lista de agencias de respuesta a la eventualidad en Haití.

El apoyo de organizaciones humanitarias con afinidad filosófica significó un aliciente para el personal asistencial del Hospital Adventista de Diquini.

El alto volumen de pacientes no solo demandaba amplitud de espacios, personal humano y tecnocientífico, sino que requería de virtudes humanas intrínsecas dentro de los equipos de respuesta.

Uno de los propósitos definidos dentro del colectivo colombiano que hizo presencia en Haití, fue el del relevo del personal asistencial, que hasta la fecha llevaban 3 días sin cese alguno de actividad médica.

La atención del paciente en ambientes prehospitalarios conlleva una preparación temática 
en espacios similares, que bien deben ser parte de la experiencia de todo grupo SAR, que da respuesta ante un evento calamitoso.

La experiencia en el manejo clínico con tecnólogos en atención prehospitalaria (TAPH) con acompañamiento médico demuestra las funcionalidades que este personal tiene en situaciones de desastre dado su solvente manejo de patologías urgentes, especialmente del trauma.

La conformación de los equipos SAR y de respuesta a desastres (equipos de salud y otros técnicos) requiere de un solvente manejo de una segunda lengua, preferiblemente el inglés dado la sinergia que es necesaria hacer con personal y agencias internacionales.

La adhesión de un políglota nativo con manejo del idioma creole posibilitó una comunicación más adecuada con la comunidad beneficiada.

Preparativos para respuesta rápida en desastres con sostenimiento y autonomía logística siempre serán necesarios e indispensables para el envío de personal humanitario.

\section{REFERENCIAS BIBLIOGRÁFICAS}

Arcos G., Castro D., Busto P. (2002). Desastres y salud pública: Un abordaje desde el marco teórico de la epidemiología. Rev. Esp. Salud Pública; 76(2): 121132. Recuperado de Disponible en: http://scielo. isciii.es/scielo.php?script $=$ sci_arttext\&pid $=\mathrm{S} 1135$ 57272002000200006\&lng=es.

Organización Panamericana de Salud (2010). Desastres. Preparativos y mitigación en las Américas. Washington. (Edición en español).

Federación Internacional de Sociedades de la Cruz Roja y de la Media Luna Roja (2009). Ginebra. Recuperado de: http://www.ifrc.org/ es/introduccion/disaster-management/sobredesastres/definicion--de-peligro/peligrosgeofisicos-terremotos/

Gellet de P. G. (2012). El cambio de paradigma: de la atención del desastre a la gestión del riesgo. Bol. Cien. Sapiens Research. Vol. 2(1)-2012 / p: 13-17. Iglesia Adventista del Séptimo Día. Recuperado de: http://www.adventistas.ec/noticias/ UE/2010/01_25_ADRA_evaluacion.ph

Insarag. (2012). Preparedness - Response. Grupo Asesor Internacional de Operaciones de Búsqueda y Rescate Guías y Metodología. Recuperado de:http://www.insarag.org/images/stories/ INSARAG_Guidelines-2012_SPA-_Read version.pdf

Médiz A. Cristófol C. Navas M. (2010). Revista Latina de Comunicación Social. Recuperado de:http:// www.revistalatinacs.org/10SLCS/actas_2010/ Cristofol.pdf.
Negrín R., Betancourt J., Sánchez MI., Monterrey H. Y. (2012). Morbimortalidad en una unidad de cuidados intensivos de un hospital de campaña en una zona de desastre. Revista Cubana MedIntens y Em. Recuperado de: http:/www.bvs.sld.cu/ revistas/mie/vol5_2_06/mie07206.htm

Noji E.K. (2000). Impacto de los desastres en la salud pública. Impacto de los desastres en la salud pública. Bogotá D.C.: Organización Panamericana de la Salud (OPS); c2000. Capítulo 1, Naturaleza de los desastres: sus características generales y sus efectos en la salud pública; 3 - 20.

Organización Panamericana de la Salud (2008). Mitos y realidades de los desastres naturales. [CD-ROM]. Washington: Program Emergency Preparedness and Disaster Relief; c2008. 1 CD-ROM: sonido, color.

Organización Panamericana de la Salud (2012). Asistencia humanitaria en caso de desastres. Guía para proveer una ayuda eficaz. Recuperado de: http://www.paho.org/spanish/ped/pedhumes.pdf.

Organización Panamericana de la Salud (2003). Gestión deresiduos sólidos en situaciones de desastres. ed. (OPS) . Washintong, D.C

RodríguezA., Terry B. (2002). Guía práctica para la fase de emergencia en caso de desastres y poblaciones desplazadas. Revista Cubana Hig Epidemiología; 2002;40(2):112.

Rodríguez A., Terry B (2005).Estrategia integral de atención e la posemergencia ante el sismo del eje cafetero colombiano. Revista Cubana Hig Epidemiología; 2005;43(2). 